\title{
Pain in thalassemia - an emerging complication
}

\author{
P.J. Giardina \\ Weill Cornell Medical Center, New York, NY, USA
}

\begin{abstract}
Many thalassemia subjects both transfused Major (TM) and nontransfused Intermedia (TI) suffer from longstanding bone disease, reduced or low bone mass (osteopenia or osteoporosis), fractures and bone pain. Unexpected musculoskeletal disease occurs despite longstanding hypertransfusion and new iron chelation strategies. Conditions which have been implicated in its pathogenesis include the massive ineffective erythropoiesis, chronic hypoxia associated with anemia, the local metabolic dysfunction from hemochromatosis, iron chelation toxicity, trace mineral deficiencies such as zinc deficiency, low vitamin D concentrations, the effect of endocrine dysfunction such as hypoparathyroidism, hypogonadism and growth hormone deficiency from hemochromatosis and the chronic inflammatory state induced by iron excess. The pathogenesis of bone disease has been attributed to the underlying marrow expansion of medullary bone caused by the massive ineffective erythropoiesis and subsequent cortical thinning. The process of normal bone health is maintained by a metabolic interplay of several hormonal factors including growth hormone, estrogen, testosterone, parathyroid hormone all of which can be diminished by iron overload in Thalassemia. Trace metals and vitamins including calcium, copper, zinc or vitamin $\mathrm{C}$ can also be deficient from iron excess or iron chelation which are also important contributors to bone metabolism. Indeed toxicities of iron chelation itself on bone development in the growing child associated with zinc deficiency, high Deferoxamine dosing and low iron burdens or the collagenous joint disease associated with deferiprone chelation have further contributed to the current musculoskeletal disease of Thalassemias. Decreased spinal height, vertebral flattening and scoliosis have also been reported. Magnetic Resonance Imaging (MRI) of adolescent and adult $\beta$ Thalassemia Major and Intermedia patients with osteoporosis and pain have assist-
\end{abstract}

Correspondence: P.J. Giardina, Weill Cornell Medical Center, New York, NY, USA.

Key words: thalassemia, pain.

(C) Copyright P.J. Giardina, 2011

Licensee PAGEPress, Italy

Thalassemia Reports 2011; 1(s2):e23

doi:10.4081/thal.2011.s2.e23

This article is distributed under the terms of the Creative Commons Attribution Noncommercial License (by-nc 3.0) which permits any noncommercial use, distribution, and reproduction in any medium, provided the original author(s) and source are credited.

Parts of this work were presented at the "12th International Conference on Thalassemia and Hemoglobinopathies", Antalya (Turkey), 11-14 May 2011. ed in defining the associated musculoskeletal pathology. The outcomes of the TCRN cross sectional observational study of low bone mass revealed the nature of bone disease across all ages and thalassemia syndromes and identified the prevalence and history of fractures and observed pain. Longstanding osteopenia and osteoporosis existed in the majority of subjects across all thalassemia syndromes. The TCRN Bone Study showed high prevalence of low BMD, fractures and bone pain in Thalassemia. A strong association between low bone mass and fractures was identified factors that may contribute to the pathogenesis of bone disease in Thalassemia. Bone mass was reduced even in children; $55 \%$ of 6 - 10 year olds had osteopenia or osteoporosis. Low vitamin D levels were also associated with increased pain in Thalassemia Major $(P=0.010)$. Pain has been associated with vitamin D deficiency and vitamin D abnormalities are prevalent in Thalassemia compared to the general population. Musculoskeletal disease of thalassemia may be related to the underlying disease, the consequences of iron excess, associated inflammatory mechanisms or iron chelator effects. Further studies are needed to identify its etiology and improve treatment strategies and prevent the emerging progressive osteoporotic disease process in thalassemia associated with high risk of fractures and musculoskeletal pain disrupting quality of life causing anxiety and depression into adolescence and adulthood.

\section{Introduction}

There is now a growing awareness that many thalassemia subjects both transfused Major (TM) and non-transfused Intermedia (TI) suffer from longstanding bone disease, reduced or low bone mass (osteopenia or osteoporosis), fractures and bone pain. The increased life span in thalassemia has allowed for the morbidities of both the underlying disease and its treatment to unfold. Unexpected musculoskeletal disease occurs despite longstanding hypertransfusion and new iron chelation strategies. ${ }^{1}$ A number of reports have examined the effect of various conditions on the pathogenesis of pain and bone disease in thalassemia but studies have been limited by small sample size and variable practice regimens. The prevalence of osteoporosis, fractures and pain across all the thalassemia syndromes has been recently documented by the North American Thalassemia Clinical Research Network (TCRN) and studied by other international groups to better understand its prevalence, severity, etiology and treatments.

Conditions which have been implicated in its pathogenesis include the massive ineffective erythropoiesis, chronic hypoxia associated with anemia, the local metabolic dysfunction from hemochromatosis, iron chelation toxicity, trace mineral deficiencies such as zinc deficiency, low vitamin D concentrations, the effect of endocrine dysfunction such as hypoparathyroidism, hypogonadism and growth hormone deficiency from hemochromatosis and the chronic inflammatory state induced by iron excess.

Historically, our understanding of musculoskeletal pain and bone disease in Thalassemia had been defined by clinical and radiographic 
findings. ${ }^{2}$ The marked osseous changes and in particular of the facial features and limb deformities with abnormal vertebral spine development, pathological fractures, and premature epiphyseal closure. ${ }^{3,4}$ The pathogenesis of bone disease has been attributed to the underlying marrow expansion of medullary bone caused by the massive ineffective erythropoiesis and subsequent cortical thinning. ${ }^{5}$

Bone is a dynamic tissue which has a resting phase and remodeling phase throughout life. Normally bone resorption, the activity of osteoclasts is balanced by bone formation the activity of osteoblasts. However, the process of normal bone health is maintained by a metabolic interplay of several hormonal factors including growth hormone, estrogen, testosterone, parathyroid hormone all of which can be diminished by iron overload in Thalassemia. Trace metals and vitamins including calcium, copper, zinc or vitamin $\mathrm{C}$ can also be deficient from iron excess or iron chelation which are also important contributors to bone metabolism.

The classic radiologic findings of thalassemia bone disease have included the hair on end appearance associated with the widened diploic space of the skull, the premature epiphyseal fusion of the humeral or femoral growth plates causing shortening of the long extremities and genu valgum; prominent vertical trabeculations of biconcave spinal vertebrae resulting in frequent vertebral compression fractures and scoliosis. ${ }^{6}$ The non-transfused or intermittently transfused thalassemia Intermedia patients still have the enhanced risk of developing extramedullary hematopoiesis with paraspinal masses that may progress and compromise the skeletal integrity of the spinal vertebrae causing cord compression, paresthesias, pain and potential paralysis. ${ }^{7}$ Prior to adequate chelation regimens synovial iron deposition in major and minor joints was also documented radiographically associated with arthralgias and arthropathies.

Hypertransfusion therapy maintains pre-transfusion hemoglobin levels at near normal levels of $\geq 9$ to $10 \mathrm{gm} / \mathrm{dl}$ was introduced nearly 40 years ago. Despite only partially correcting the anemia and incompletely suppresseing the massive ineffective erythropoiesis, hypertransfusion is successful in improving or preventing the disfiguring boney deformities of thalassemia. Iron chelation with subcutaneous prolonged infusions of deferoxamine was also begun nearly 40 years ago. Therefore the early reports of osteoporosis with low bone mass in regularly transfused and well chelated thalassemic adolescent and adult subjects by dual energy absorptometry (DXA) was unexpected. ${ }^{1}$ Indeed toxicities of iron chelation itself on bone development in the growing child associated with zinc deficiency, high Deferoxamine dosing and low iron burdens or the collagenous joint disease associated with deferiprone chelation have further contributed to the current musculoskeletal disease of Thalassemias. ${ }^{8,9}$ Decreased spinal height, vertebral flattening and scoliosis have also been reported. ${ }^{10}$

The availability of radiographic DXA measurements of bone mineral content and density as well as magnetic resonance skeletal imaging have increased our understanding of the musculoskeletal disease in Thalassemia.

Magnetic Resonance Imaging (MRI) of adolescent and adult $\beta$ Thalassemia Major and Intermedia patients with osteoporosis and pain have assisted in defining the associated musculoskeletal pathology. Our series of cervical and thoracolumbar MRI studies in these patients complaining of neck, back or hip pain, MRI findings included 54\% with degenerative disc disease, 34\% disc bulging, 29\% disc herniation, 7\% spinal stenosis, $10 \%$ arthritis and $4 \%$ vertebral compression fractures.

The outcomes of the TCRN cross sectional observational study of low bone mass revealed the nature of bone disease across all ages and thalassemia syndromes and identified the prevalence and history of fractures and observed pain. ${ }^{11}$ This study consisted of 371 thalassemia subjects including $\beta$ Thalassemia Major, Intermedia, $\beta$ E Thalassemia, Hb $\mathrm{H}$ Disease and $\mathrm{Hb} \mathrm{H}$ Constant Spring Disease who ranged from 6 to 75 years with $49 \%$ male. Longstanding osteopenia and osteoporosis existed in the majority of subjects across all thalassemia syndromes. The greater age, hypogonadism, increased bone turnover and lower weight were independent indicators of low bone mass regardless of the thalassemia syndrome. (Vogiatzi JMBR 2009) Bone and joint pain 30 days prior to enrollment was reported in $34 \%$ of subjects in whom $6 \%$ required prescription pain medications, and $12.2 \%$ used over the counter analgesics. By gender, $48 \%$ of females but only $28 \%$ of males complained of recent pain. The odds of severe pain increased by $47 \%$ for each 5 year age increment. Pain was reported more frequently in $\beta$ TM at $40 \%$ in comparison to $\beta$ TI at $16 \%$ and $\beta \mathrm{E}$ at $19 \%$.

The sites of pain included back pain in $24 \%$, head and neck in $10 \%$, shoulder, hip and legs in $8 \%$, pain in the hands and feet in 5\%. The pain duration averaged 3 days per week and lasted on average 7.5 hours per day. $34 \%$ of subjects reported that their transfusions reduced or eliminated the pain. However, the pain increased by $47 \%$ for each 5 year age increment.

The overall fracture rate was $26 \%$ in the TCRN Study and $8.9 \%$ of these subjects reported 3 or more lifetime fractures. The cumulative risk of fractures increased linearly with age. No sex difference in fracture rate was observed except for those male subjects $<20$ years of age were more likely than females to experience fractures. Mean ratio $2.5 \%, 95 \mathrm{CI}, 1.1-5.6(\mathrm{p}=0.026)$. Subjects with $\beta \mathrm{E}$ Thalassemia had decreased fracture rates compared to $\beta$ TM. Fractures among adults was $55 \%$ in TM and $71 \%$ in TI. The site of fractures was variable. Extremity fractures were most commonly found in $30 \%$ and vertebral and hip fractures were reported in $3.6 \%$. The increased fracture rates were independently associated with lower spine $\mathrm{Z}$ scores and relative weight. Sex hormone replacement therapy was a significant predictors of fracture risk. For each 1.5 decrease in spine $\mathrm{Z}$ score, the fracture rate increased by $37 \%$. Recent bone or joint pain was reported in all subjects with a history of fractures.

The TCRN Bone Study showed high prevalence of low BMD, fractures and bone pain in Thalassemia. A strong association between low bone mass and fractures was identified factors that may contribute to the pathogenesis of bone disease in Thalassemia. Subjects with hypogonadism, hepatitis $\mathrm{C}$ and heart disease with high bone turnover had a higher likelihood of osteoporosis. Bone mass was reduced even in children; $55 \%$ of 6 - 10 year olds had osteopenia or osteoporosis. There was a strong negative association between bone mass and age in $11-19$ year olds. Peak bone mass was suboptimal in adolescence which is a critical period for the development of low bone mass in Thalassemia owing to their enhanced risk of hypogonadism or delayed puberty requiring hormone replacement therapy. Trials to target Thalassemia children and adolescents to indentify the role of gondal steroids, growth hormone and vitamin D supplementation are of priority to improve bone accrual in Thalassemias.

To determine the prevalence, predictors and effects of pain in Thalassemia major the TCRN Thalassemia Longitudinal Cohort (TLC) Study evaluated 265 adolescent and adult patients aged 14 to 58 years, mean age $29.4 \pm 10.4 \mathrm{yrs}, 52 \% \mathrm{~F}$ and $103 \beta$ TM children aged 5 to 14 years (mean $9.8 \pm 2.6 \mathrm{yrs}$ ) $52 \%$ F who reported pain severity on the SF$36 \mathrm{~V} 2 \mathrm{QOL}$ survey and as parents reporting on the PF-28CHQ. ${ }^{12,13}$

Overall $69 \%$ reported bodily pain in the past month; $28 \%$ of adults reported at least moderate pain; $50 \%$ reported pain that interfered with work and $25 \%$ reported moderate interference with work. Parents reported pain fairly often in their children in the past month. Pain severity increased with age on the SF-36 Pain scale. Quality of life due to pain in thalassemia adults declined greatly with age compared only to a slight decline in the general population. Quality of life did not decline significantly in children with Thalassemia compared to the general population. Increased bodily pain was significantly correlated with increased anxiety $(\mathrm{r}=-0.37)$ and depression $(\mathrm{r}=-0.36)(\mathrm{p}<$ $0.001)$. Low vitamin D levels were also associated with increased pain 
in Thalassemia Major $(\mathrm{p}=0.010) .{ }^{12}$ Pain has been associated with vitamin $\mathrm{D}$ deficiency and vitamin $\mathrm{D}$ abnormalities are prevalent in Thalassemia compared to the general population. Vitamin D levels are deficient $(<11 \mathrm{ng} / \mathrm{ml})$ in $12 \%$ adolescents with Thalassemia, insufficient $(11-30 \mathrm{ng} / \mathrm{ml})$ in $69 \%$ and only normal (> $30 \mathrm{ng} / \mathrm{ml})$ in $18 \%$ of adolescent subjects in the TCRN low bone mass study. ${ }^{14}$

In a cohort of 232 transfusion dependent subjects TM and E $\beta$ Thal subjects $56 \% \mathrm{~F}$ who previously reported at least mild pain prospectively completed a daily questionnaire during 3 consecutive transfusion cycles addressing the prevalence and severity of pain. High prevalence of pain was associated with age. Pain reduction was more pronounced in those following transfusion especially those transfused at $>4$ week intervals. $^{12}$

\section{Conclusions}

In conclusion, the pathogenesis of musculoskeletal pain and bone disease is still not completely clear and its prevention and treatment are unresolved. Musculoskeletal disease of thalassemia may be related to the underlying disease, the consequences of iron excess, associated inflammatory mechanisms or iron chelator effects. Further studies are needed to identify its etiology and improve treatment strategies and prevent the emerging progressive osteoporotic disease process in thalassemia associated with high risk of fractures and musculoskeletal pain disrupting quality of life causing anxiety and depression into adolescence and adulthood.

\section{References}

1. Giardina PJ, Schneider R, Lesser M, et al. Abnormal bone metabolism in thalassemia. In endocrine disorders in thalassemia, S. Ando and C. Brancati (eds.) Springer Verlag Berlin Heidelberg New York, 1995:39-46.

2. Caffey I. Cooley's Anemia: a review of the roentgenographic find- ings in skeleton. Am JR 78:381-391.

3. Dines DM, Canale VC, Arnold WD. Fractures in thalassemia. J Bone Joint Surg Am. 1976;58:662-6.

4. Currarino G, Erlandson ME. Premature fusion of epiphyses in Cooley's anemia. Radiology. 1964;83:656-64.

5. Piomelli S, Danoff SJ, Becker MH, Lipera MJ, Travis SF. Prevention of bone malformations and cardiomegaly in Cooley's anemia by early hypertransfusion regimen. Ann N Y Acad Sci. 1969;165:42736.

6. Papageorgiou 0, Papanastasiou DA, Beratis NG, Korovessis P, Oikonomopoulos A. Scoliosis in $\beta$ thalassemia. Pediatrics. 1991;88:341-5.

7. Kaufman T, Coleman M, Giardina P, Nisce LZ. The role of radiation therapy in the management of hematopoietic neurologic complications in thalassemia. Acta Haematol; 1991;85:156-159.

8. Al-Refaie FN, Wonke B, Hoffbrand AV. Arthropathy in thalassaemia patients receiving deferiprone. Lancet. 1994;344:262-3.

9. Brill PW, Winchester P, Giardina PJ, Cunningham-Rundles S. Desferrioxamine-induced bone dysplasia in patients with thalassemia major. AJR Am J Roentgenol. 1991;156:561-5.

10. Papageorgiou 0, Papanastasiou DA, Beratis NG, Korovessis P, Oikonomopoulos A. Scoliosis in $\beta$ thalassemia. Pediatrics. 1991;88:341-5.

11. Vogiatzi MG, Macklin EA, Fung EB, et al.; for the Thalassemia clinical research network. Bone disease in thalassemia: A frequent and still unresolved problem. J Bone Miner Res. 2009;24:543-57.

12. Trachtenberg F, Foote D, Martin M, et al.; for the Thalassemia clinical research network. Pain as an emergent issue in thalassemia. Am J Hematol. 2010;85:367-370.

13. Sobota A, Yamashita R, Xu Y, et al.; for the Thalassemia clinical research network: Quality of life in thalassemia: A comparison of SF-36 results from the thalassemia longitudinal cohort to reported literature and the US norms. Am J Hematol. 2010 Sep 30.

14. Vogiatzi MG, Macklin EA, Trachtenberg FL, et al.; for the Thalassemia clinical research network. Differences in the Prevalence of growth, endocrine and vitamin D abnormalities among the various thalassemia syndromes in North America. Br J Haematol. 2009;146:546-56. 